\title{
Clostridium symbiosum
}

National Cancer Institute

\section{Source}

National Cancer Institute. Clostridium symbiosum. NCl Thesaurus. Code C86296.

A species of anaerobic, Gram positive, rod shaped bacteria assigned to the phylum

Firmicutes. This species is sporeforming, motile by flag ella, indole negative,

nonhemolytic, does not hydrolyze esculin and produces acid from glucose, mannose,

fructose and galactose. C. symbiosum is an emerging pathogen but rarely isolated from clinical specimens. 